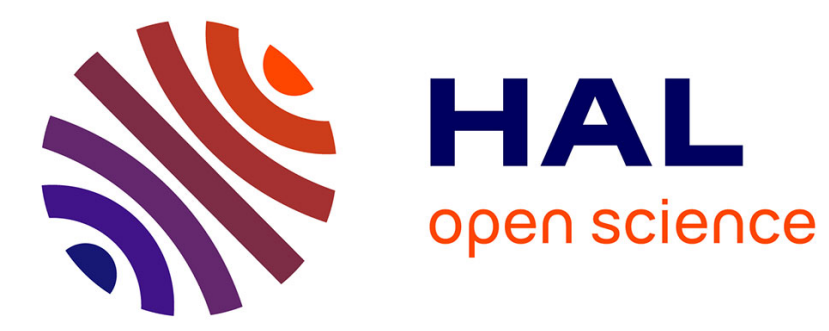

\title{
Post-machining distortion mitigation via bending straightening, a multiparametric ROM study
}

\author{
Ramiro Mena, Jose Aguado, Stéphane Guinard, Antonio Huerta
}

\section{To cite this version:}

Ramiro Mena, Jose Aguado, Stéphane Guinard, Antonio Huerta. Post-machining distortion mitigation via bending straightening, a multiparametric ROM study. Proceedings of the 22nd International ESAFORM Conference on Material Forming: ESAFORM 2019, May 2019, Vitoria-Gasteiz, Spain. 10.1063/1.5112642 . hal-02454607

\section{HAL Id: hal-02454607 https://hal.science/hal-02454607}

Submitted on 24 Jan 2020

HAL is a multi-disciplinary open access archive for the deposit and dissemination of scientific research documents, whether they are published or not. The documents may come from teaching and research institutions in France or abroad, or from public or private research centers.
L'archive ouverte pluridisciplinaire HAL, est destinée au dépôt et à la diffusion de documents scientifiques de niveau recherche, publiés ou non, émanant des établissements d'enseignement et de recherche français ou étrangers, des laboratoires publics ou privés. 


\title{
Post-machining distortion mitigation via bending straightening, a multiparametric ROM study
}

\author{
Ramiro Mena ${ }^{1,2,3, a)}$, José V. Aguado ${ }^{2}$, Stéphane Guinard ${ }^{1}$ and Antonio Huerta ${ }^{3}$ \\ ${ }^{1}$ Airbus SAS, France \\ ${ }^{2}$ Institut de Calcul Intensif ICI-HPC at École Centrale de Nantes, France \\ ${ }^{3}$ Laboratori de Calcul Numeric (LaCaN). Departament de Matematica Aplicada III. E.T.S. de Ingenieros de \\ Caminos, Canales y Puertos, Universitat Politecnica de Catalunya, Spain

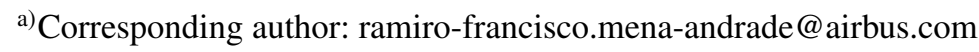

\begin{abstract}
Large and thick aeronautical structural parts frequently exhibit significant distortions after machining because of the residual stresses generated along the manufacturing process. A post-machining shape correction phase, called reshaping, has to be applied to each part before proceeding to the assembly stage. At the moment this process relies entirely on the know-how and experience of a specialized operator. As a consequence, the process is expensive and time-consuming. Therefore, there is a need at an industrial level to solve this problem with the support of numerical simulation tools. However, to reach this target, it is required to explore beforehand the input space to construct a solution dictionary in order to provide real-time response. Both requirements oblige to launch a multi-parametric analysis under a Model Order Reduction (MOR) framework. A complete reshaping process consists of a sequence of elementary operations, among which bending straightening is selected in its three-point bending configuration and it is simulated by applying the Sparse Subspace Learning method (SSL). By using the MOR approach, new insights for the distortion mitigation via plastic bending can be explored that will help to understand the open problem of reshaping.
\end{abstract}

\section{INTRODUCTION}

Large and thick aeronautical structural parts are made of aluminum forgings in order to produce a lightweight construction [1]. However, they frequently exhibit significant distortions after machining because of the residual stresses generated during the sequential manufacturing steps, especially after the heat treatment of quenching [2, 3]. Such distortions are variable in nature, but the industrial process incorporates a robust reshaping stage: skilled boilermakers operate series of mechanical loadings on the non-conforming part and re-establish its nominal geometry while obeying guidelines imposed by stress offices - which guarantees the produced part has still mechanical characteristics compatible with its operational role - Nonetheless, numerical simulations may offer here huge margins for improvements: operational assistance to boilermakers and related gains in time-cycle (optimized reshaping sequence, optimized parameters for each elementary reshaping operation), improved knowledge of actual part and related gains in safety (monitoring of actual plastic strains) $[4,5]$. An example of the structural parts subjected to reshaping are the cruciform beams that form the wing-box in an aircraft.

Reshaping can be considered as the sum of four local operations where the part is plastically deformed. These operations are: bending straightening, torsion straightening, roller burnishing, and ultrasonic needle peening. The main idea in this process is to produce a local deformation in the opposite direction of the deformed shape in order to counteract the mismatch between the real and the theoretical geometry.

This corrective stage is an iterative operation and its work-flow diagram is represented in Figure 1. Starting from the initial geometry, a measurement and control step is performed in order to determine the level of distortion. Based on the result, one of the four reshaping operations described before is selected. To perform one single plastic deformation step, the selected reshaping operation needs to be configured, it means, the technological parameters $\mu_{i}$ (e.g position, imposed displacement, length of the application) need to be defined. This process is repeated until the part is repaired and fits into the design tolerance. 


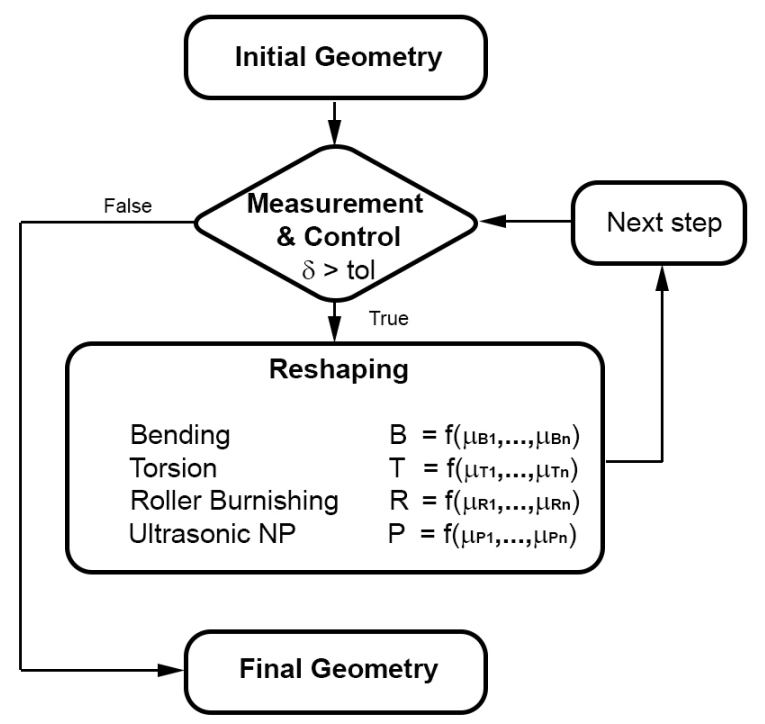

FIGURE 1. Reshaping work-flow.

As stated before, nowadays, reshaping relies on the experience of a well-trained operator. The goal to introduce numerical simulation in this process is to assist the operators and provide support during the decision-making step in order to reduce the number of operations needed to repair a part. However, from the technical point of view, reshaping simulation presents three main challenges:

- the initial distortion after machining is unknown and, with the available state-of-the-art simulation techniques, there is a mismatch between the numerical and the real distortion [6].

- distortions vary from one part to the other (non reproducibility), that is why reshaping cannot be standardized. As a consequence, reshaping simulation needs to provide multiple possible initial distortion scenarios.

- $\quad$ each time a new reshaping operation is performed, distortion changes and with it, the technological parameters associated with the next reshaping operation. Hence, reshaping simulation must be able to handle multiple parameters in real-time.

Under the described scenarios, the requirement of multiple reshaping simulations together with a real-time response obliges to launch a multiparametric analysis under a MOR framework [7]. For the different MOR available techniques, the Sparse Subspace Learning (SSL) is the selected method to study reshaping thanks its non-intrusiveness and the capability to handle the nonlinearities present during the process (e.g geometrical non-linearity and plasticity). For a detailed description of the method, the interesed reader can be referred to $[8,9]$.

This document is organized as follows: first, the SSL formulation applied to reshaping is explained. Then, the problem setup used for a T shaped beam geometry is described. Next, the results of the multi-parametric ROM study are analysed and finally, the obtained conclusions are addressed.

\section{RESHAPING UNDER THE SSL FORMULATION}

Sparse Subspace Learning (SSL) is a non-intrusive Model Order Reduction technique, suitable for multiparametric studies and nonlinear analysis. The method exploits the sparsity of the solution with respect to the parameters in order to build a parametric solution from the computation of only few wisely chosen snapshots [8]. From the four available reshaping techniques, the bending straightening operation is selected in its three-point bending configuration to be studied with the SSL method, due to this operation is the most used to repair the structural parts in the industry [10]. 
For bending straightening, we are interested in determining the final shape of a part after performing a cycle of loading and unloading. Therefore, the displacement field $\mathbf{u}(\mathbf{x})$ for a given set of parameters $\boldsymbol{\mu}$ is our objective function and, by using the separated variable representation under the SSL formulation, it can be written as:

$$
\mathbf{u}(\mathbf{x} ; \boldsymbol{\mu})=\sum_{i=0}^{k} \alpha_{i}(\mathbf{x}) \psi_{i}(\boldsymbol{\mu})
$$

where $\alpha(\mathbf{x})$ and $\psi(\boldsymbol{\mu})$ are defined as the surpluses and parametric functions, respectively. The parametric functions $\psi(\boldsymbol{\mu})$ act as the shape function. They are known, define the hierarchical basis and correspond to the Lagrangian polynomials, while the surpluses functions $\alpha(\mathbf{x})$ need to be determined. They are calculated as a linear combination of sampling or measurements evaluated at the collocation points $\mathcal{P}_{n}=\left\{\mu_{0}, \mu_{1}, \ldots ., \mu_{n}\right\}$.

For a unidimensional parametric problem, the collocation points are the extreme of the $n$-th Chebyshev polynomials of the first kind, $T_{n}(\mu)$ and are referred as Gauss-Chebyshev-Lobatto points (GCL) too.

In order to handle a multidimensional parametric problem, first, each parameter is treated as the unidimensional case described before and then, the Smolyak rule is introduced with the aim of constructing the sparse grid to be used during sampling. To put into perspective the advantage of the sparse sampling respect to the full sampling based on the tensor product, for a 2 parameters problem and a selected hierarchy level $k=5$, the full sampling requires $33^{2}=1089$ points, while the sparse sampling only needs 177 points, providing a reduction of $83.7 \%$ in terms of computational cost.

Once the sampling points are defined, the simulations are launched in a commercial FEM software (e.g ABAQUS, PamCrash). The displacement field $\mathbf{u}\left(\mathbf{x} ; \mu_{i}\right)$ is known at each collocation point and for our case, it is considered as the reference solution. With this available information, the corresponding surplus functions are determined as the difference between the exact and the approximated solution at the hierarchy level $(k-1)$, as expressed in the following expression:

$$
\alpha_{i}(\mathbf{x})=\mathbf{u}\left(\mathbf{x} ; \mu_{i}\right)-\mathbf{u}^{k-1}\left(\mathbf{x} ; \mu_{i}\right)
$$

Coming back to the reshaping problem, for the three-point bending straightening operation, the set of parameters $\boldsymbol{\mu}$ is composed by the initial distortion $\delta_{0}$, the position of the bottom roller $r_{b}$ and the imposed vertical displacement $y_{s}$. For our problem, each parameter is considered as a dimension.

$$
\boldsymbol{\mu}=\left(\delta_{0}, r_{b}, y_{s}\right)
$$

When the solution $\mathbf{u}(\mathbf{x} ; \boldsymbol{\mu})$ is reconstructed for all the parametric domain, the remaining distortion $\delta$ can be found as a post-processing operation and it is defined as:

$$
\delta(\boldsymbol{\mu})=\max _{\mathbf{x}}[\mathbf{u}(\mathbf{x} ; \boldsymbol{\mu})]-\min _{\mathbf{x}}[\mathbf{u}(\mathbf{x} ; \boldsymbol{\mu})]
$$

However, for all the possible reshaping configurations, we are mainly interested to know the optimum stroke $y_{s}^{o p t}$ that, for a fixed set of parameters $\left(\delta_{0}, r_{b}\right)$ will minimize the remaining distortion $\delta$ in the structural part.

$$
y_{s}^{o p t}=\underset{y_{s}}{\arg \min }\left[\delta\left(\delta_{0}, r_{b}, y_{s}\right)\right]
$$

\section{PROBLEM SETUP}

To study the three-point bending operation, a $\mathrm{T}$ shaped beam is defined, as shown in Figure 2 . This geometry corresponds to a simplified version of a cruciform part, which exhibits critical distortions when machined and is a primary structure of main importance for aircraft manufacturers. The parameters to be explored are available in Table 1 with is minimum and maximum values $\mu_{\min }$ and $\mu_{\text {max }}$, respectively.

The selected material is an aluminum AA7010 previously characterized by Airbus Group Innovations. The elastic material properties are summarized in Table 2. To run the reshaping simulations, a Chaboche's isotropic/nonlinear kinematic hardening model was used. However, as the material parameters are under a nondisclosure agreement, they are not included in this document.

Finally, a Python script in combination with ABAQUS was used to run the 123 required simulations to explore the parametric input space under the SSL method for a hierarchy level k=3. 


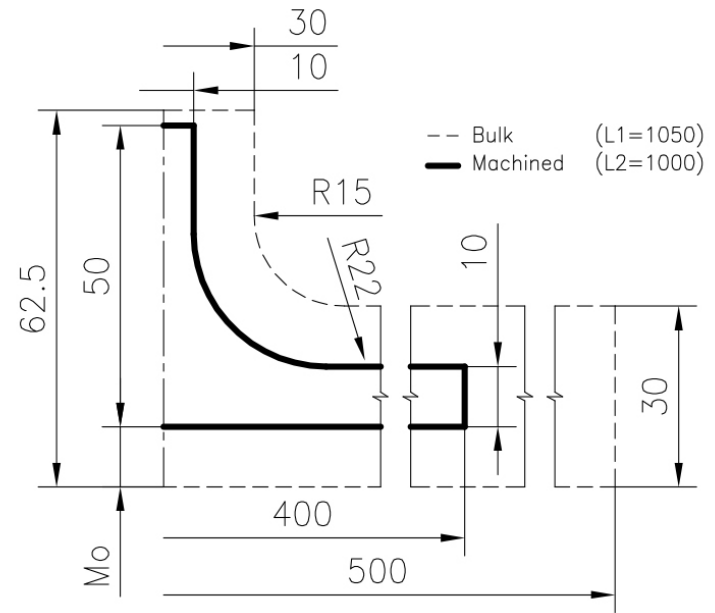

(a)

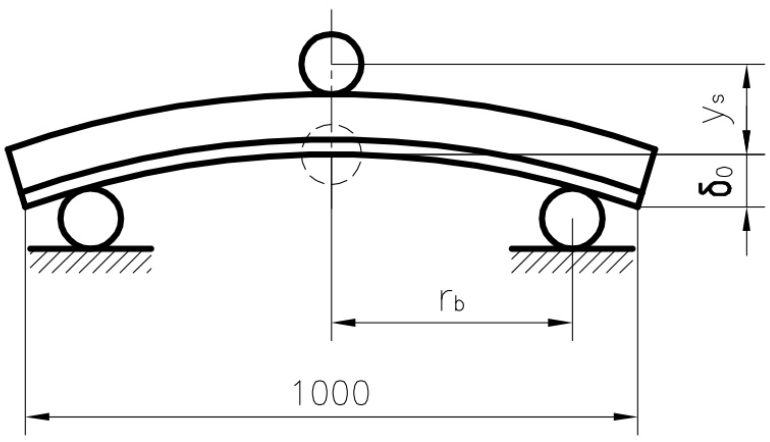

(b)

FIGURE 2. $T$ shaped beam. a) Geometry definition and b) Parameters representation for three-point bending straightening.

TABLE 1. Parameters values for the three-point bending operation

\begin{tabular}{clcc}
\hline Parameter ID & \multicolumn{1}{c}{ Description } & $\mu_{\min }$ & $\mu_{\max }$ \\
\hline 1 & Initial distortion $\delta_{0}(\mathrm{~mm})$ & -15 & 0 \\
2 & Bottom roller position $r_{b}(\mathrm{~mm})$ & 100 & 485 \\
3 & Imposed vertical displacement $y_{s}(\mathrm{~mm})$ & 5 & 30
\end{tabular}

TABLE 2. Elastic properties for Aluminum AA7010

\begin{tabular}{ccc}
\hline Young's Modulus E (MPa) & Poisson's ratio $\boldsymbol{v}(-)$ & Yield stress $\sigma_{\boldsymbol{y}}(\mathbf{M P a})$ \\
\hline 73000 & 0,3 & 390
\end{tabular}

\section{RESULTS AND DISCUSSION}

Before proceeding to explain the effect of each parameter in the bending straightening operation, it is required to introduce the evolution of the remaining distortion $\delta$ along the process. An example of the different zones present during reshaping is represented in Figure 3. In the beginning, if a small value of $y_{s}$ is applied, the warped geometry will remain inside of the elastic region and there is no modification of $\delta_{0}$. We define this region as zone $\mathrm{A}$ and it is characterized by the relation $\delta \approx \delta_{0}$. A reduction of the initial distortion is only performed when plasticity takes place along the part. We call zone $\mathrm{B}$ at the region where $\delta<\delta_{0}$. Inside this zone, the optimum configuration $\left(\delta=\delta_{\text {min }}\right)$ is located in a narrow space. To find properly this configuration is one of the challenges of reshaping. On the other hand, an excessive value of $y_{s}$ can be counter-productive and invert the shape of the initial distortion from concave to convex (or vice-versa), giving as a result the operation zone $\mathrm{C}$, where $\delta>\delta_{\min }$, and which should be avoided.

The reshaping diagram described before is the result of a particularized solution where $\delta_{0}$ and $r_{b}$ were previously defined and $y_{s}$ was set free. In order to generalize the problem, the next step is to set free a pair of parameters (e.g $\delta_{0}$ and $y_{s}$ ), giving, as a result, a response surface. With the aim to explore the obtained multi-parametric solution, instead of using a 3D representation, a 2D plot together with level curves are used to describe if the remaining distortion $\delta$ will decrease or increase for a fixed bottom roller position $r_{b}$, as can be appreciated in Figure 4 .

Effect of the initial distortion $\delta_{0}$ : In a previous work, it has been proved that reshaping can be studied by using as a main input $\delta_{0}$ under a Residual Stress Free hypothesis (RSF) [5]. This approach was used in order to generate numerically the warped geometry. By including $\delta_{0}$ as a parameter, the problem of not knowing its magnitude beforehand is overcome. In that way, the reshaping problem is solved numerically for all the interval of initial distortions and 


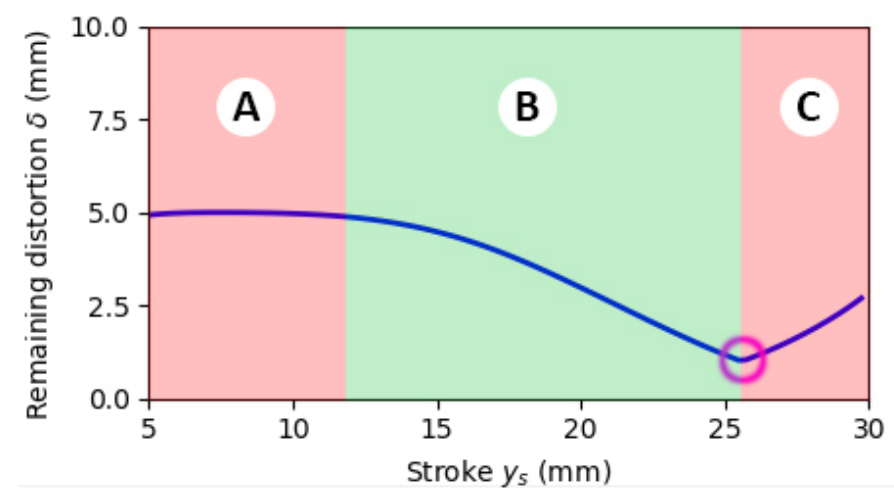

Zones:

A. No reshaping is performed $\delta=\delta 0$

B. Reshaping takes place $\delta<\delta 0$

- Optimum configuration $\delta=\delta$ min

C. Distortion increment $\quad \delta>\delta$ min

FIGURE 3. Zones present during reshaping as a function of the stroke $y_{s}$.

once the part is machined, the operator only needs to access to the solution dictionary and select a distorted geometry closer to the experimental result.

Effect of the bottom roller position $r_{b}$ : Once the initial distortion $\delta_{0}$ is known, the next step is to select the reshaping operation. For the three-point bending operation, the internal roller (top) is fixed and located at the middle of the beam while the external rollers (bottom) need to be placed. The position of the bottom roller is defined by the parameter $r_{b}$ and it defines the slope of the response surface that relates the remaining distortion $\delta$ with $\delta_{0}$ and $y_{s}$.

A value of $r_{b}$ closer to the top roller produces a difficult reshaping operation to control, as it is expected to have a quick change of the remaining distortion $\delta$ during bending. On the other hand, a value of $r_{b}$ away from the top roller generates the opposite behavior with a progressive variation of $\delta$. In the performed parametric study, the selected value of $r_{b}$ particularizes a page of the solution dictionary.

Effect of the imposed vertical displacement $y_{s}$ : For a fixed initial distortion and a selected bottom roller position, the three-point bending operation is completed when a vertical displacement $y_{s}$ is imposed at the internal roller. The magnitude of $y_{s}$ needs to be defined in order to minimize the remaining distortion $\delta$. The response surface obtained for a fixed $r_{b}$ shows that reshaping is more difficult to be performed for smaller values of $\delta_{0}$ as the required stroke $y_{s}$ only to induce plasticity in the cross section is bigger than $\delta_{0}$. On the other hand, for a bigger magnitude of $\delta_{0}$, it is expected to have more margin to find the stroke that minimizes $\delta$.

\section{CONCLUSIONS}

The industry is willing to have new tools to solve problems that nowadays are treated in an empirical way. Reshaping of large thick structural parts is an example of how MOR can be applied to an open problem, especially the SSL, allows to perform a multi-parametric study to test different what-if scenarios in a cost-effective way and, thanks to its non-intrusiveness, acts as a bridge to join MOR with the available third-party FEM solutions.

For the case of bending straightening, the initial distortion $\delta_{0}$ is the starting point for reshaping and it determines the setup for the technological parameters $r_{b}$ and $y_{s}$. Additionally, the problem of not knowing beforehand the value of $\delta_{0}$ is overcome by including it as a parameter. Then, thanks to the SSL, this reshaping operation can be studied in a virtual environment before launching the real process.

Future work will focus on how to chain multiple reshaping operations and to include material properties as extra parameters. As expected, the computational cost for the numerical analysis will increase. Again, here it resides the importance of studying reshaping with MOR techniques. 


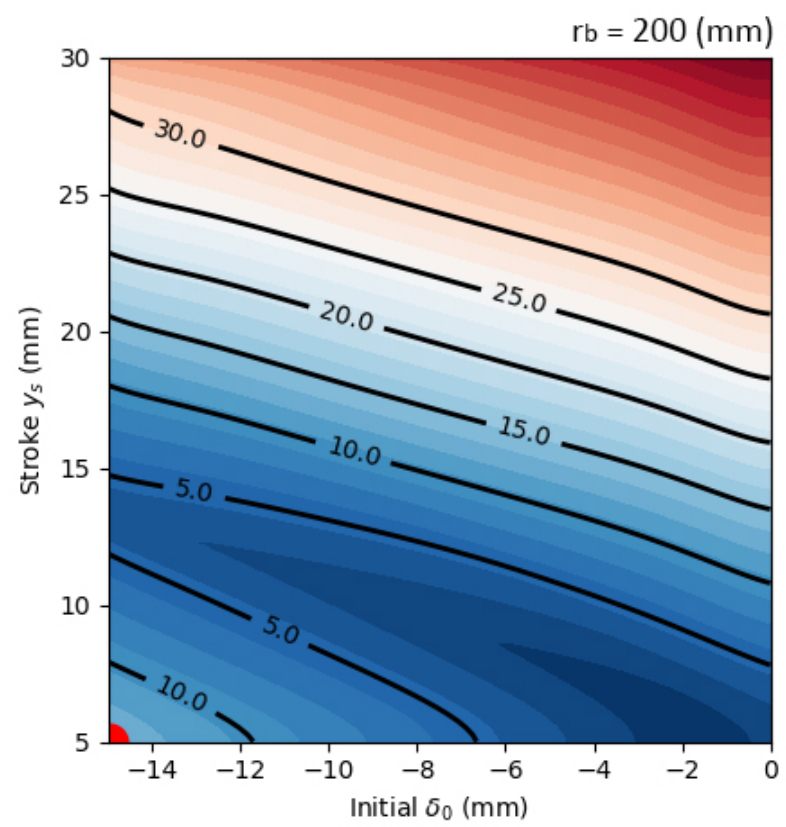

(a)

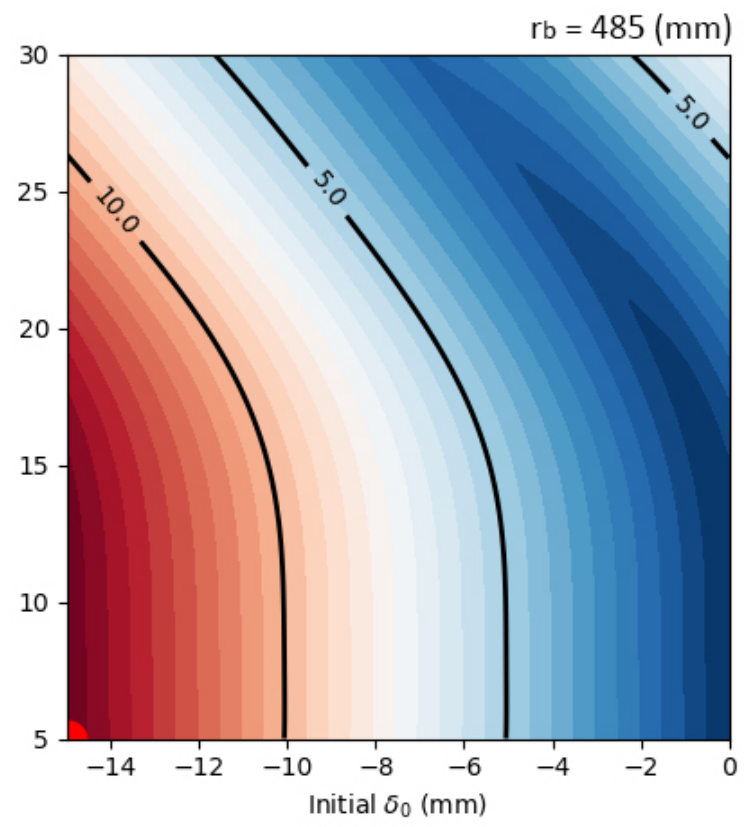

(b)

FIGURE 4. Response surface: Remaining distortion $\delta$. Solution selection as a function of $r_{b}$. a) $r_{b}=200(\mathrm{~mm})$ and b) $r_{b}=485(\mathrm{~mm})$.

\section{ACKNOWLEDGMENTS}

This project is part of the Marie Skłodowska-Curie ITN-ETN AdMoRe funded by the European Union Horizon 2020 research and innovation program with grant number 675919.

\section{REFERENCES}

[1] M. Kleiner, M. Geiger and A. Klaus, CIRP Annals, 52 (2003) 521-542.

[2] K. Ma, R. Goetz, and S. K. Srivatsa, "Modeling of Residual Stress and Machining Distortion in Aerospace Components," Tech. Rep. (Air Force Research Laboratory, 2010)

[3] J.S. Robinson, S. Hossain, C.E. Truman, A.M. Paradowska, D.J. Hughes, R.C. Wimpory and M.E. Fox, Mater. Sci. Eng. A., 527 (2010) 2603-2612.

[4] W.-M. Sim, "Challenges of residual stress and part distortion in the civil airframe industry," in 2nd International Conference on Distortion Engineering (IDE) (2008).

[5] R. Mena, D. Deloison, J. V. Aguado, and A. Huerta, "Influence of the residual stresses in reshaping operations of large aeronautical parts," in International Conference on Adaptive Modeling and Simulation ADMOS 2017, edited by S. Perotto and P.Diez (CIMNE, 2017).

[6] X. Cerutti, "Numerical modelling and mechanical analysis of the machining of large aeronautical parts : Machining quality improvement," Ph.D. thesis, Ecole Nationale Superieure des Mines de Paris 2014.

[7] F. Chinesta, A. Leygue, F. Bordeu, J. V. Aguado, E. Cueto, D. Gonzalez, I. Alfaro, A. Ammar, and A.Huerta, Arch Computat Methods Eng (2017)., 20 (2013) 31-59.

[8] D. Borzacchiello, J. Aguado and F. Chinesta, Arch Computat Methods Eng (2017)

[9] D. Borzacchiello, J. Aguado and F. Chinesta, Int J Numer Method H, 27 (2017) 1602-1622.

[10] M. Mitze, HTM, 2 (2010) 110-117. 